\title{
Dissimilarity of microbial diversity of pond water, shrimp intestine and sediment in Aquamimicry system
}

Shenzheng Zeng ${ }^{1+} \mathbb{0}$, Sukontorn Khoruamkid ${ }^{2 \dagger}$, Warinphorn Kongpakdee ${ }^{2}$, Dongdong Wei ${ }^{1,3}$, Lingfei $\mathrm{Yu}^{1}$, Hao Wang ${ }^{1,3}$, Zhixuan Deng ${ }^{1}$, Shaoping Weng ${ }^{1,3,4}$, Zhijian Huang ${ }^{1,3,4^{*}}$, Jianguo He $\mathrm{e}^{1,3,4^{*}}$ and Kriengkrai Satapornvanit ${ }^{2^{*}}$

\begin{abstract}
The Pacific white shrimp, with the largest production in shrimp industry, has suffered from multiple severe viral and bacterial diseases, which calls for a more reliable and environmentally friendly system to promote shrimp culture. The "Aquamimicry system", mimicking the nature of aquatic ecosystems for the well-being of aquatic animals, has effectively increased shrimp production and been adapted in many countries. However, the microbial communities in the shrimp intestine and surrounding environment that act as an essential component in Aquamimicry remain largely unknown. In this study, the microbial composition and diversity alteration in shrimp intestine, surrounding water and sediment at different culture stages were investigated by high throughput sequencing of $16 \mathrm{~S}$ rRNA gene, obtaining 13,562 operational taxonomic units (OTUs). Results showed that the microbial communities in shrimp intestine and surrounding environment were significantly distinct from each other, and 23 distinguished taxa for each habitat were further characterized. The microbial communities differed significantly at different culture stages, confirmed by a great number of OTUs dramatically altered during the culture period. A small part of these altered OTUs were shared between shrimp intestine and surrounding environment, suggesting that the microbial alteration of intestine was not consistent with that of water and sediment. Regarding the high production of Aquamimicry farm used as a case in this study, the dissimilarity between intestinal and surrounding microbiota might be considered as a potential indicator for healthy status of shrimp farming, which provided hints on the appropriate culture practices to improve shrimp production.
\end{abstract}

Keywords: Pacific white shrimp, Aquamimicry system, Microbial community, Water, Sediment, Intestine

\footnotetext{
*Correspondence: Isshzhj@mail.sysu.edu.cn; Isshjg@mail.sysu.edu.cn;

ffiskks@ku.ac.th

+'Shenzheng Zeng and Sukontorn Khoruamkid contributed equally to this work

${ }^{1}$ State Key Laboratory of Biocontrol/Southern Marine Sciences and Engineering Guangdong Laboratory (Zhuhai), School of Marine Sciences, Sun Yat-Sen University, Guangzhou, China

${ }^{2}$ Department of Fisheries, Faculty of Fisheries, Kasetsart University, Bangkok, Thailand

Full list of author information is available at the end of the article
}

\section{Keypoints}

The novel Aquamimicry system has successfully improved shrimp production.

Microbial community in shrimp intestine and environment are investigated.

Relation between intestinal and environmental microbiota indicates culture condition.

\section{Introduction}

Aquaculture remains an important source of food and nutrition for millions of people worldwide, which plays an essential role in meeting the urgent global food 
demand (Low et al. 2017). Pacific white shrimp, Litopenaeus vannamei, with the largest production in global shrimp industry, has suffered enormous economic losses due to serious infectious diseases (Zeng et al. 2020). Diseases caused by viruses and bacteria have been major impact on the sustainable development of the industry, especially the occurrence of white spot syndrome virus (WSSV) (Tassanakajon et al. 2018). Recently, newer diseases have emerged, such as early mortality syndrome (EMS), acute hepatopancreatic necrosis disease (AHPND) or hepatopancreas necrosis syndrome (HPNS) (Flegel 2019; Huang et al. 2016), and white feces syndrome (WFS) (Huang et al. 2020; Zeng et al. 2020). These diseases occurred in many Asian countries including China, India, Indonesia, Thailand and Vietnam. As previously reported, inappropriate aquaculture practices can lead to elevated levels of persistent organic pollutants, metals parasites and viruses in aquaculture field (Amir et al. 2008). Hence, good aquaculture practices should be the main focus of shrimp farming to increase shrimp immunity against diseases and avoid the deterioration of water quality during culture period. Lately, a number of researches have indicated that balance of the microbial community plays a major role in the disease occurrence (Biesebeke 2018; Butto and Haller 2016). Hence, there is a need to develop reliable, repeatable and environmentally friendly technology to combat new and existing pathogens.

The "Aquamimicry System" was established in 2013 by Mr. Sutee Prasertmark and Mr. Veerasan Prayotamornkul, who are long-time shrimp farmers in Thailand. The combination of nature condition and technology leads to more sustainable shrimp farming practices by mimicking the natural aquatic environment. Chemicals used is avoided by employing the symbiotic system, which is created through prebiotics (non-digestion component but can be metabolized by specific microorganism) and probiotics, defined as living microorganisms that have a positive effect on host (Biesebeke 2018; Butto and Haller 2016). The success of this system is based on the production of the natural food for shrimp resulting in the decrease in feed conversion ratio, maintenance of optimal water and sediment quality by microorganisms, and disease elimination (Biesebeke 2018; Butto and Haller 2016). This expertise has been presented to a large number of shrimp producers, and is now adapted in many countries, such as Australia, Bangladesh, Brazil, Brunei, China, Ecuador, Egypt, India, Korea, Malaysia, Mexico, Peru, Singapore, Sri Lanka, USA, and, Vietnam.

As part of this novel shrimp culture technique, the microbial communities of the shrimp intestine and surrounding environment must be studied. Previous studies indicated that shrimp sourced little of their intestinal microbiota from their rearing water, whereas the successions of intestinal microbiota were significantly correlated with host age (Xiong et al. 2019, 2020). Indeed, identification of the microbial community present in the shrimp and nearby environment at different culture stages could facilitate our understanding of the main and important microbial groups which will help to prevent disease outbreaks and maintain good water quality condition during the culture period.

This study investigated the diversity of intestinal microbial community in Pacific white shrimp, sediment, and culture water in a shrimp farm in Nakhon Nayok province, Thailand. This was done by analyzing the types and relationships among the microbial community in sediment, water and shrimp intestines, the relationship of microbial community in shrimp intestine and the surrounding environment, and the microbial alterations at different culture periods. The research results can support the promotion to use of the probiotic and Aquamimicry system in sustainable aquaculture.

\section{Materials and methods Sample collection}

The Aquamimicry shrimp farm is located in Nakhon Nayok province, central region of Thailand $\left(14.14^{\circ} \mathrm{N}\right.$, $100.99^{\circ}$ E). From August to October 2018, samples were collected every 15 days from 15 days post-hatching from four shrimp pond (named as A, B, C and D). Each pond has an area of $4000 \mathrm{~m}^{2}$, and $1.5 \mathrm{~m}$ depth. Shrimp were cultured at a stocking density ranging from 160,000 to 200,000 shrimps per pond $\left(40-50 \mathrm{shrimp} / \mathrm{m}^{2}\right)$. Probiotics were applied during the culture period, such as Bacillus subtilis, Bacillus amyloliquefaciens, Bacillus valismortis, Bacillus megaterium and Bacillus licheniformis.

Twenty shrimp samples per pond were collected. After measuring length and weight, each shrimp's surface was separately sterilized with $70 \%$ ethanol and the intestine was aseptically dissected into $2 \mathrm{~mL}$ sterilized tube containing $1 \mathrm{~mL}$ DNA preservation solution.

Water samples were also obtained from three different locations in each pond, with $500 \mathrm{~mL}$ water per location. Each water sample was then put in ice separately before filtering through $0.22 \mu \mathrm{m}$ polyethersulfone filter membranes with a vacuum pump. For the chemical analyses, $200 \mathrm{~mL}$ of each sample was collected from the same location using sterile bottles.

Three replicates of sediment samples were collected with $500 \mathrm{~mL}$ sterile bottle from different locations. For each sample, $50 \mathrm{~g}$ of sediment was used for total nitrogen (TN) and total phosphate (TP) determination, and $10 \mathrm{~g}$ of sediment stored into $50 \mathrm{~mL}$ sterilized tube for DNA extraction. All the samples were stored at $-20{ }^{\circ} \mathrm{C}$ before DNA extraction and quality determination. 


\section{Water and sediment quality determination}

Water quality parameters such as temperature, $\mathrm{pH}$, dissolved oxygen (DO), and salinity were measured on-site using a YSI handheld multi-parameter instrument (Model YSI 380, YSI Incorporated, Ohio, USA). $\mathrm{TN}$, dissolved inorganic nitrogen $\left(\mathrm{NH}_{4}{ }^{+}-\mathrm{N}, \mathrm{NO}_{2}{ }^{-} \mathrm{N}\right.$, and $\left.\mathrm{NO}_{3}{ }^{-}-\mathrm{N}\right), \mathrm{TP}$, and orthophosphate $\left(\mathrm{PO}_{4}{ }^{3-}-\mathrm{P}\right)$ were measured using an automatic discrete analyzer (Model CleverChem 380, DeChem-Tech, Hamburg, Germany). Sediment $\mathrm{pH}$ was measured in situ with a $\mathrm{pH}$ meter (Model SX630, Sanxin, Shanghai, China).

\section{DNA extraction and sequencing}

Intestine and sediment genomic DNA were extracted by the QIAamp PowerFecal DNA Kit (Qiagen, Dusseldorf, Germany), whereas water DNA was extracted by the MinkaGene Water DNA Kit (mCHIP, Shenzheng, China). The concentration and purity of genomic DNA were measured using the NanoDrop One Spectrophotometer (Thermo Fisher, Massachusetts, USA). The primer pair 515F (5'-GTGCCAGCMG CCGCGGTAA$\left.3^{\prime}\right)$ and 806R (5'-GGACTACHVGGGTWTCTAAT-3') were used to amplify the V4 region of $16 \mathrm{~S}$ rRNA gene, which was modified with a barcode tag containing a random 6-base oligos.

PCR products was then purified with EZNA Gel Extraction Kit (Omega, Georgia, USA). Sequencing libraries were generated using NEBNext Ultra DNA Library Prep Kit for Illumina (New England Biolabs, Massachusetts, USA) following manufacturer's recommendations. The library quality was assessed on the Qubit 2.0 Fluorometer (Thermo Fisher, USA) and Agilent Bioanalyzer 2100 system (Agilent, California, Germany). The libraries were then mixed in equidensity and sent for sequencing by a Hiseq2500 platform (Illumina, California, USA), which was conducted by Guangdong MAGIGENE Biotechnology Co., Ltd. (Guangzhou, China). Raw data generated from Hiseq2500 platform were paired-end reads.

\section{Bioinformatics and statistical analysis}

To control the sequencing quality, raw tags with low quality (quality value $<20$, sequence length $\leq 100 \mathrm{bp}$ ) were filtered by Trimmomatic (Version 0.33, https:// www.usadellab.org/cms/? page $=$ trimmomatic). To merge reads of the same DNA fragment, FLASH (Version 1.2.11, https://ccb.jhu.edu/software/FLASH/) was used to gain the splicing sequences (Magoč and Salzberg 2011). Based on the unique barcode, sequences were assigned to samples and then removed off the barcode and primer sequence by Mothur (Version 1.35.1, https://www.mothur.org) (Schloss et al. 2009).

Sequences with more than $97 \%$ similarity were assigned as the same operational taxonomic units (OTU) (Edgar 2013). The chimera sequence and singleton OTU were removed during the OTU clustering by USEARCH (Version 10, https://www.drive5.com/usearch/) (Edgar 2010). For each representative sequence, the SILVA database (Release 132, https://www.arb-silva.de/) was used annotate taxonomic information with the confidence threshold to default to $\geq 0.5$. The OTUs abundance information was normalized using a standard of sequence number corresponding to the sample with the least sequences.

Alpha diversity, including Shannon index and Simpson index were calculated with the QIIME (Version 1.9.1, https://qiime.org/) (Caporaso et al. 2010). Bray-Curtis distance was used to evaluate the species complexity differences of samples. Principal coordinate analysis (PCoA) was conducted to reveal the clustering of samples using the vegan package in $\mathrm{R}$ (Version 3.6.0). Random forests regression was used to regress relative abundances of taxa in the temporal profiles of water, intestine and sediment samples, using the following parameters with randomForest package in $R$ ( $c$. fold $=10$, step $=0.99$, ntree $=5000$ ). To perform the clustering of OTUs for the analysis of time-course data, the fuzzy c-means algorithm was used for the comparative clustering analysis with Mfuzz package in R (Israel et al. 2016). Permutational multivariate analysis of variance (PerMANOVA) was conduct to compare microbial composition dissimilarities (Anderson 2006). A calculated $P$ value $<0.05$ was considered statistically significant. To evaluate the correlation between environmental factors and microbial community, variation partition analysis (VPA) canonical correlation analysis (CCA) was conducted using the vegan package in $R$.

\section{Results}

\section{Shrimp production and environmental factors}

The average shrimp length and weight data every 15 days of culture are shown in Additional file 1. Tables S1 and S2. At harvest, the average weights ranged from 23.8 to $25.6 \mathrm{~g}$, production ranged from 3872.6 to $3980 \mathrm{~kg} / \mathrm{hec}-$ tare, and survival rates were 95, 100, 82 and $90 \%$ for ponds $\mathrm{A}, \mathrm{B}, \mathrm{C}$ and $\mathrm{D}$, respectively. The feed conversion ratio ranged from 1.31 to 1.4 , and shrimps were sold at USD 6.38 per kg (Table 1 ).

Water quality parameters were determined throughout the shrimp culture. Temperature was relatively stable at $28-29{ }^{\circ} \mathrm{C}$. Average $\mathrm{pH}$ values were between 7.45 and 8.0 (Fig. 1). Weekly salinity gradually decreased during culture from 2-5 (Fig. 1). Likewise, the average dissolved oxygen value decreased from $5.875 \mathrm{mg} \cdot \mathrm{L}^{-1}$ to 
Table 1 The information of pond production

\begin{tabular}{lllll}
\hline Details & \multicolumn{4}{l}{ Pond no } \\
\cline { 2 - 5 } & A & B & C & D \\
\hline Pond size $\left(\mathrm{m}^{2}\right)$ & 4,000 & 4,000 & 4,000 & 4,000 \\
Culture period (days) & 72 & 72 & 70 & 73 \\
Initial stocking & 160,000 & 160,000 & 200,000 & 180,000 \\
Stocking density (shrimp/ $\left.\mathrm{m}^{2}\right)$ & 40 & 40 & 50 & 45 \\
Count (shrimp/kg) & 39 & 41 & 41 & 42 \\
Harvest size (g) & 25.6 & 24.4 & 24.4 & 23.8 \\
Shrimp harvest (kg) & 3900.0 & 3900.0 & 3980.0 & 3872.6 \\
Total feed used (kg) & 5091.8 & 5241.8 & 5053.5 & 5427.5 \\
Survival rate (\%) & 95 & 100 & 82 & 90 \\
Feed conversion rate & 1.31 & 1.34 & 1.27 & 1.4 \\
Prize (US \$/kg) & 6.38 & 6.38 & 6.38 & 6.38 \\
Total income (US \$) & $24,879.3$ & $24,879.3$ & $25,389.7$ & $24,704.5$ \\
\hline
\end{tabular}

$4.113 \mathrm{mg} \cdot \mathrm{L}^{-1}$ (Fig. 1). Average concentrations of $\mathrm{NH}_{3}-\mathrm{N}$, $\mathrm{NO}_{2}-\mathrm{N}, \mathrm{NO}_{3}-\mathrm{N}$ and $\mathrm{PO}_{4}{ }^{3-}$ throughout the culture period were between $0.522-1.841 \mathrm{mg} \cdot \mathrm{L}^{-1}, 0.006-0.2516 \mathrm{mg} \cdot \mathrm{L}^{-1}$, 0.0117-0.7587 $\mathrm{mg} \cdot \mathrm{L}^{-1}$ and $0.0359-0.3453 \mathrm{mg} \cdot \mathrm{L}^{-1}$, respectively (Fig. 1). Sediment $\mathrm{pH}$ value ranged between 5.6 and 6.7 (Fig. 1).

\section{Microbial composition and diversity in Aquamimicry system}

Quality and chimera filtration of the raw data produced a total of 21,657,470 high quality sequencing reads from 162 samples, with an average of 133,688 reads. Finally, 13,562 OTUs were obtained, and the OTUs numbers detected in each sample ranged from 888 to 4,399 , with an average of 2190 OTUs. OTUs were identified into 62 phyla and 2203 genera. Sequences that could not be classified into any known groups were assigned as "others".

In the three habitats, Proteobacteria, Bacteroidetes, Planctomycetes, Firmicutes, Actinobacteria and Cyanobacteria were the dominant phyla. At phylum level, Proteobacteria was the most abundant in water $(20.8 \%)$ and intestine (43.6\%), while Bacteroidetes was dominant in sediment (32.1\%) (Additional file 1. Fig. S1). At the genus level, the most abundant genera were Exiguobacterium, Vibrio, Candidatus Bacilloplasma, Pirellula, Pseudarthrobacter, Acinetobacter, Rhodopirellula and Photobacterium (Additional file 1. Fig. S2).

To estimate and compare the bacterial diversity in the three habitats, $\alpha$-diversity indices (Chao1 and Shannon index) were calculated from OTUs of each sample. The Shannon indices of water, intestine and sediment were $5.2 \pm 1.0,5.6 \pm 0.8$ and $8.9 \pm 0.3$ respectively (Fig. 2a). The Chaol indices of water, intestine and sediment were $1,673 \pm 495,1,320 \pm 336$ and $4,038 \pm 287$ respectively
(Fig. 2b), which suggested that the sediment microbial community held the highest $\alpha$-diversity.

\section{Microbial similarities and differences among the three habitats}

For further investigation of the dominant microbiota that exists in the three habitats, a Venn diagram revealed that 4,831 OTUs were shared in shrimp intestine, surrounding water and sediment (Fig. 3a). These OTUs hold a large percentage of the total bacterial population (average of $81.5,85.7$ and $76.5 \%$ in water, intestine and sediment, respectively). In addition, unique OTUs in three habitats were observed, such as $224,1,861,2,439$ unique OTUs were only found in water, intestine and sediment, respectively. PCoA analysis revealed that the microbial communities of the three habitats were markedly distinct from each other (Fig. 3b).

To find out the specific bacterial taxa distributed among the three habitats, we constructed a random forest classifier model that could specifically identify samples in each group. To detect unique OTU markers, a ten-fold cross-validation on a random forest model with all 162 samples was conducted. Results indicated that a total of 23 OTU markers were selected as the optimal marker set. The relative abundance of these OTUs in each group was presented (Fig. 3c), which were further proven to be significantly different among the three habitats by ANOVA $(P<0.05)$ (Fig. 3d). Compared to the surrounding water and sediment, some opportunistic pathogens (Vibrio, Aeromonas, Photobacterium and Candidatus Bacilloplasma) were significantly sufficient in the intestine $(P<0.05)$. The UPGMA clustering showed that almost all the individual samples were clustered into groups according to habitat (Fig. 3e), which suggested that the 23 OTU markers were a successful in distinguishing the specific taxa in each habitat.

\section{Microbial alterations during different culture periods}

The difference in microbial communities at different culture stages was further investigated. In the three habitats, PCoA results showed that all the individual samples were clustered into groups according to stage of culture (Fig. 4a). PERMANOVA $(P<0.05)$ confirmed that the microbial communities differed significantly between any two of the compared stages $(P<0.05)$, except between stages 2 and $3(P>0.05)$ (Table 2$)$.

For further determination of which variables affected the shrimp intestinal microbial structure, a VPA was conducted. A sunset of environmental parameters explained $9.0 \%$ of the observed microbial variation, while the effect of different culture stages was more pronounced $(11.2 \%)$ (Additional file 1. Fig. S3a). CCA was applied to reveal the correlation between environmental parameters and 

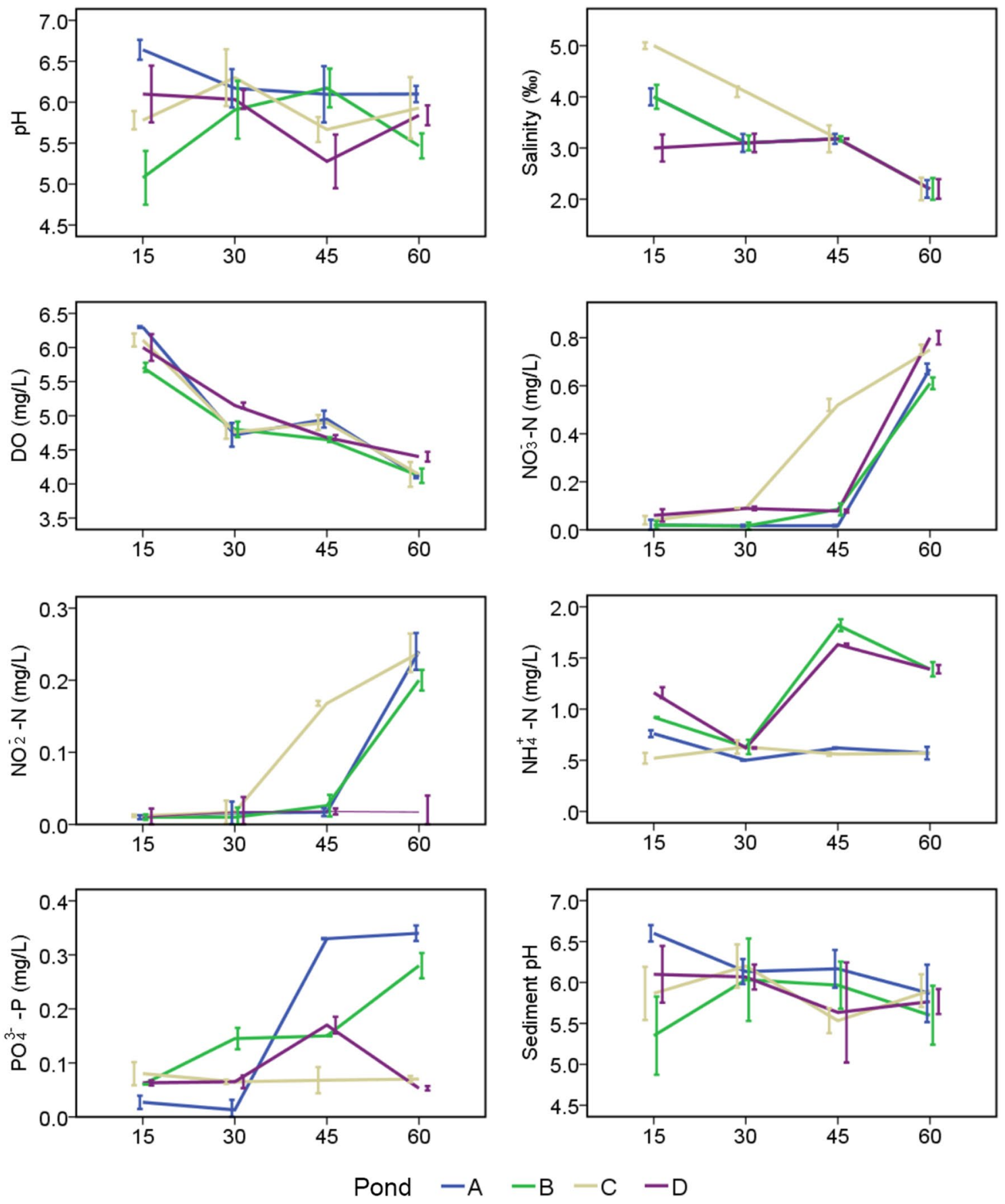

Fig. 1 The physicochemical factors of the water and sediment

microbial community (Additional file 1. Fig. S3b). Salinity, water $\mathrm{pH}$ and $\mathrm{DO}$ were correlated to microbial community, which indicated that they were the crucial factors shaping the variations in microbial community.

To identify the altered taxa at different stages and how the relative abundance altered, a comparative clustering strategy was implemented to detect substantial differences in abundance profiles across stages. The fuzzy c-means clustering grouped similar abundance profiles into 3, 4 and 6 clusters in water, intestine and sediment, respectively, representing distinct phases of abundance during the culture period (Fig. 4b). This strategy 

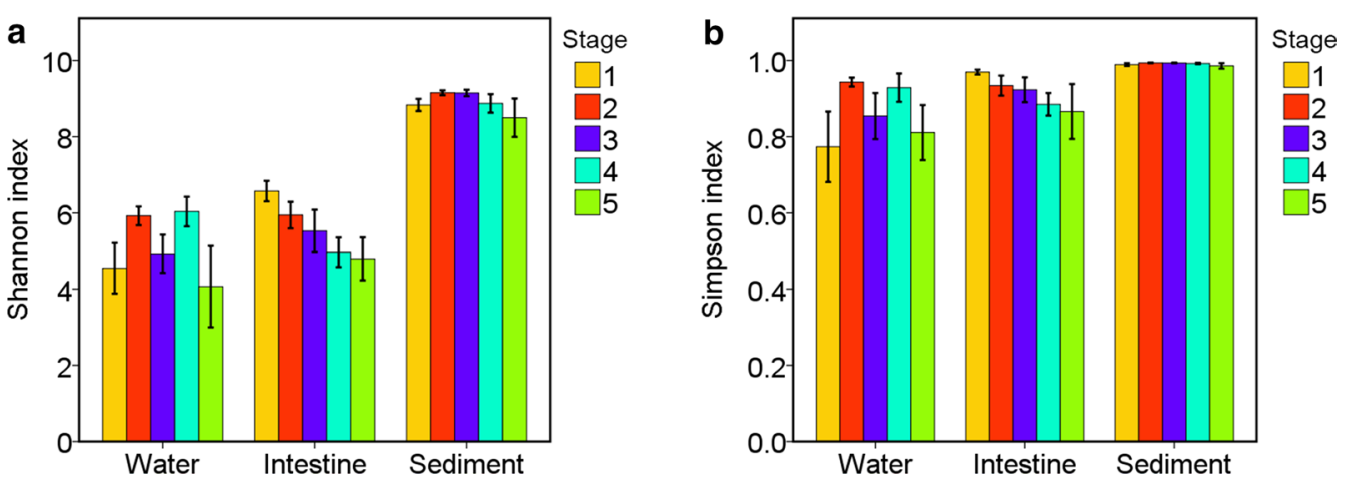

Fig. 2 The $a$-diversity comparison among three habitats. a The Shannon index $\mathbf{b}$ The Chao 1 index. Compared to water and intestine samples, the a-diversity of sediment microbiota was the highest
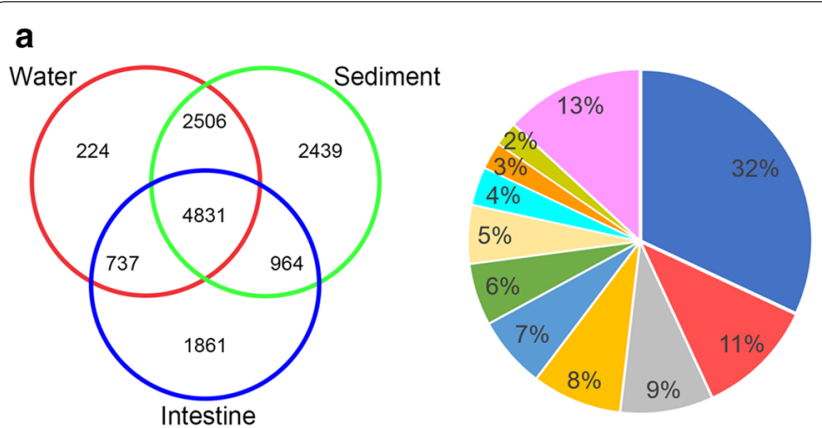

Phylum

- Proteobacteria

Bacteroidetes

Patescibacteria

Planctomycetes

- Firmicutes

- Chloroflexi

Actinobacteria

Verrucomicrobia

- Cyanobacteria

- Acidobacteria

Others

\section{b}

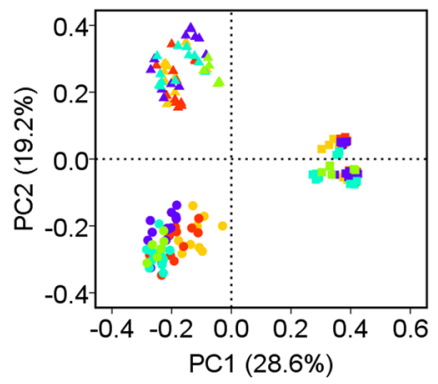

C

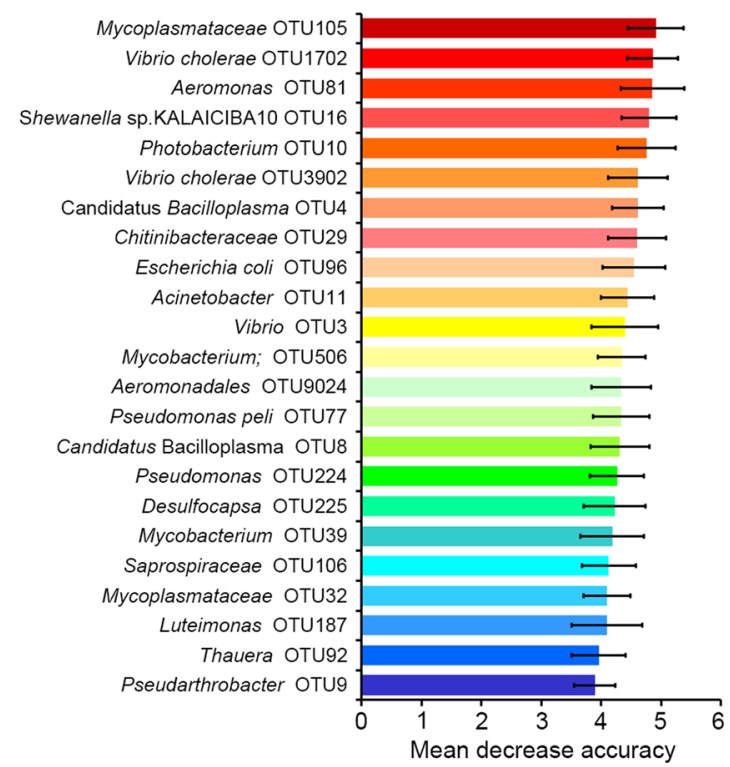

d

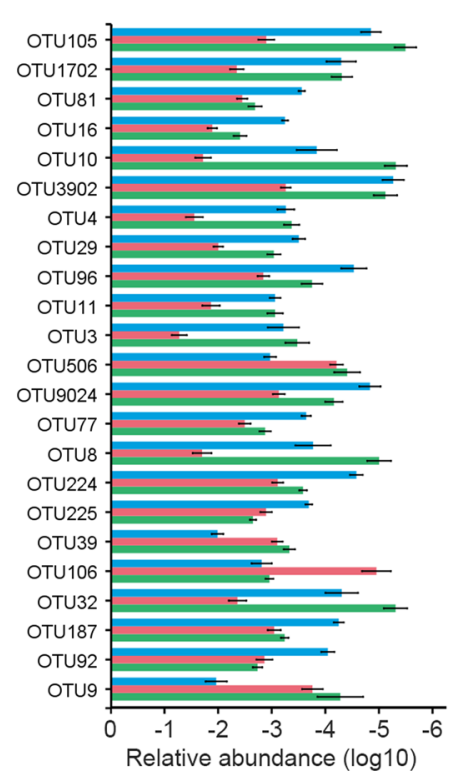

e

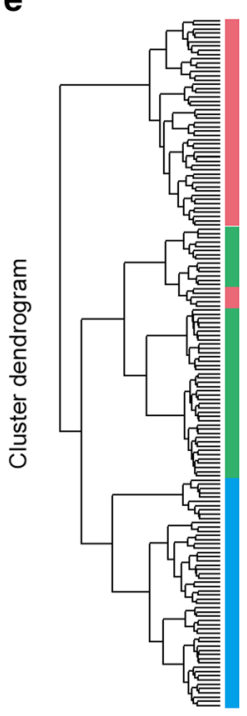

Fig. 3 The relationship between shrimp intestinal and environmental microbiota. a Venn diagram shows the unique and shared OTUs in the different habitats. the shared OTUs majorly belonged to Proteobacteria and Bacteroidetes phylum. $\mathbf{b}$ PCOA of microbial communities based on the 165 rRNA sequencing profiles. Samples were clustered into three groups by PCoA based on Bray-Curtis distance, indicating that the microbial structure differed significantly among three habitats. c Classification of the OTU markers for the three habitats. A total of 23 OTU markers were selected as the optimal marker set by a ten-fold CV-error curve. $\mathbf{d}$ The relative abundance of the OTU markers at each group. e UPGMA clustering based on the 23 markers revealed that the microbial composition of each group was clearly distinct 
(See figure on next page.)

Fig. 4 The altered microbial structure at different culture stages. a PCOA of the microbial structure at different culture stages. b OTUs with similar abundance alteration at different stages clustered into different groups. All the OTUs that were presented in more than $50 \%$ samples at each habitat were used for Mfuzz analysis. The x-axis represents culture stage, and the $y$-axis represents standardized expression change. OTUs in the same cluster suggested that their alteration trends during the culture period were similar. The Venn diagram showed the shared OTU number between similar clusters

allowed us to easily identify which taxa exhibited the same abundance patterns. Results showed that a great amount of OTUs (water: 328; sediment: 1228; intestine: 346) exhibited a dramatic alteration during the culture period, which corresponded to the results of PCoA and PERMANOVA analysis that microbial composition varied significantly at different culture stages. Moreover, the shared OTUs in the same alteration pattern was observed. There were 22 (15\%), 19 (25\%) and 21 (17\%) shared OTUs between intestine and sediment in clusters 1 to 3 , while 48 (33\%) and 7 (0.9\%) shared OTUs were found between intestine and water (Fig. 4b), which indicated that the intestinal microbial alteration did not correlated much to water and sediment during the culture period.

\section{Discussion}

A number of studies suggest that shrimp intestinal microbiota is closely related to rearing environmental microbiota, which is also linked to shrimp disease occurrence (Hou et al. 2018b; Xiong et al. 2018a). The Aquamimicry system provides an ecological pattern to prevent disease outbreaks in shrimp culture. However, little is known about the microbial composition and diversity alterations of this system in shrimp intestine and the surrounding environment. In this study, the relationship of microbial community between shrimp intestine and the surrounding environment was investigated, and the altered microbes during the culture period were further identified. This work partially meets the urgent need for understanding the main and important microbial community in Aquamimicry system.

Many studies report that the interactions of intestinal microbiota and surroundings were associated with aquatic animal diseases (Li et al. 2017b; Xiong et al. 2018a). Thus, observation of the overlap between shrimp intestinal microbiota and that of their surrounding water and sediment is necessary for shrimp health management in aquaculture. A previous study on shrimp earthen ponds demonstrated that similar bacterial community compositions were observed in shrimp intestine, the surrounding water and sediment (Hou et al. 2018b). Moreover, some reports have shown similar microbial community in intestine and surroundings of crucian carp ( $\mathrm{Li}$, et al. 2017a). However, unlike in these previous researches, this study revealed that microbial communities of the three habitats were markedly separate (Fig. 3). Furthermore, the shared OTU numbers were relatively low between the same alteration pattern of intestinal microbiota and surrounding microbiota (Fig. 4), which indicated that the microbial community in shrimp intestine was not the same as those in the surrounding water and sediment in the Aquamimicry system. A previous study exhibited similar changes between water and intestinal microbiota during the culture period, suggesting that the water microbiota altered the shrimp intestinal microbiota, and was subsequently related to disease outbreak (Xiong, et al. 2018a). The same phenomenon was found in crucial carp suffered from red-operculum disease that a close link between intestinal and environmental microbiota (Li, et al. 2017a). Similar results were found in a tilapia farm that pond water and sediment bacteria influenced the composition of intestine, especially the presence of pathogens, such as Streptococcus and Vibrio (Al-Harbi and Uddin 2005). Further study demonstrated that tilapia suffered from infections caused by an emerging Francisella sp. which was found in sediment and water with high abundance (Soto, et al. 2009). As the Pacific white shrimp is a typical benthos that takes in feed debris or sediment from the pond bottom, some culture operations in the Aquamimicry system, such as removal the feed from the bottom and using multiple oxygen increasing device to agitate the water thoroughly, can effectively reduce shrimp's intake of debris, which might weaken the effect of the environmental harmful factors on shrimp. As previously reported, shrimp sourced little of their intestinal microbiota from their rearing water, whereas the successions of intestinal microbiota were significantly correlated with host age (Xiong et al. 2019, 2020). In the present study, the culture stages were more pronounced than environmental factors in microbial variations (Additional file 1. Fig. S3), while the shared OTUs at the same stages were low (Fig. 4), which indicated that both culture stages and aquaculture pattern affected shrimp intestinal microbial composition. Taken all above clues into consideration, the divergence between shrimp intestinal and environmental microbiota in the Aquamimicry system may be a potential indicator for the healthy condition of shrimp culture, which give us inspiration on the sustainable shrimp culture practice. 

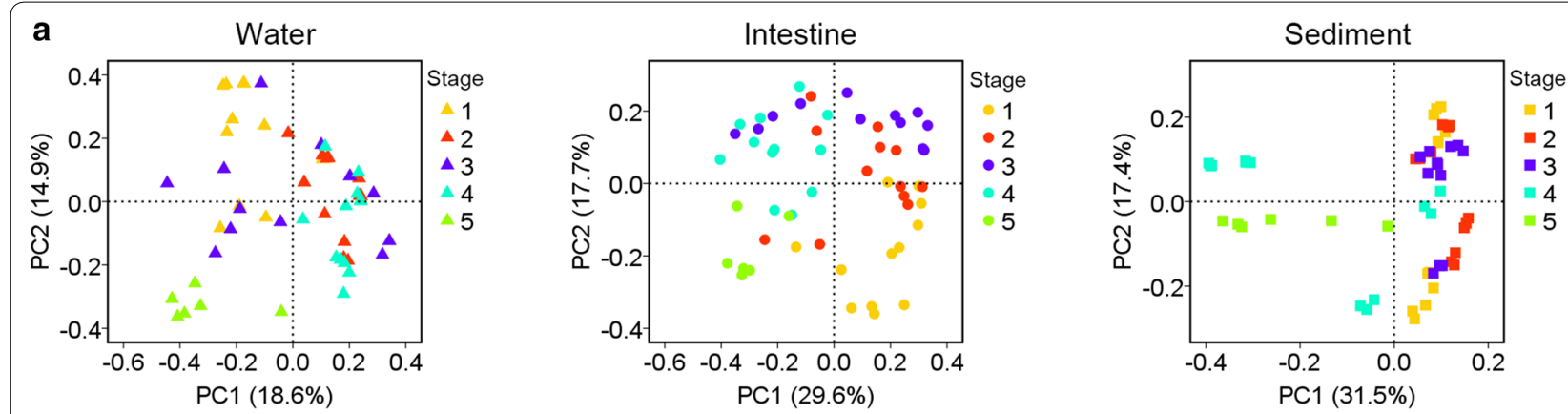

b

Water
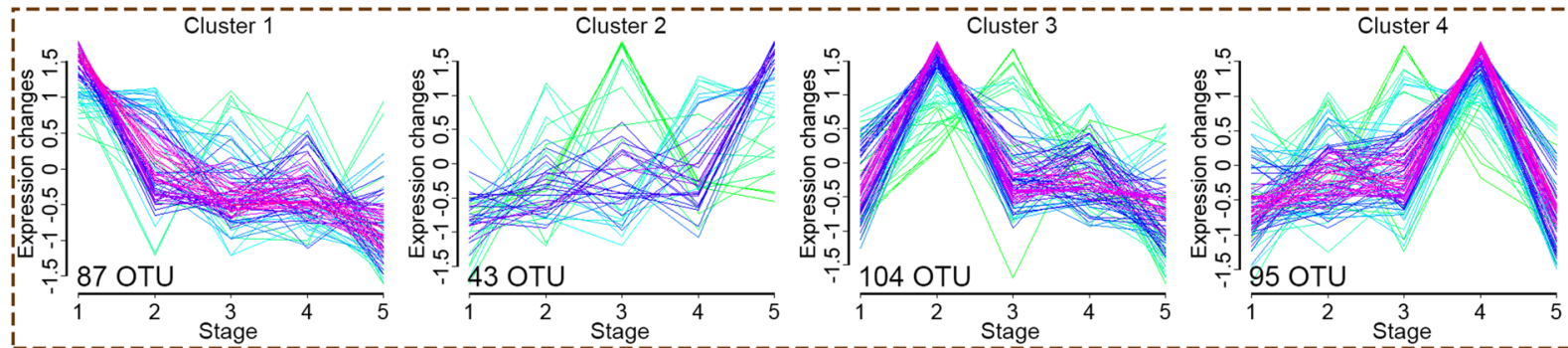

\section{Intestine}
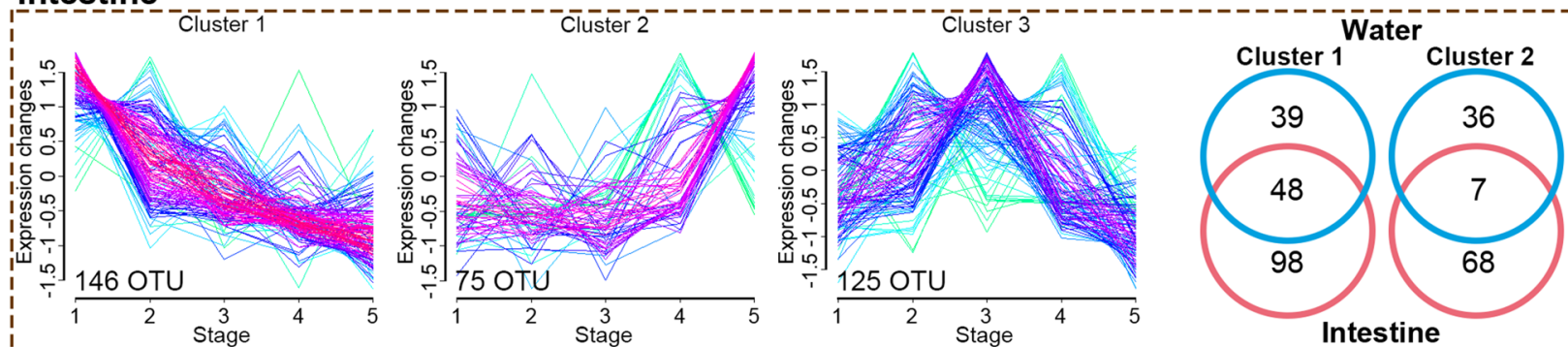

Sediment
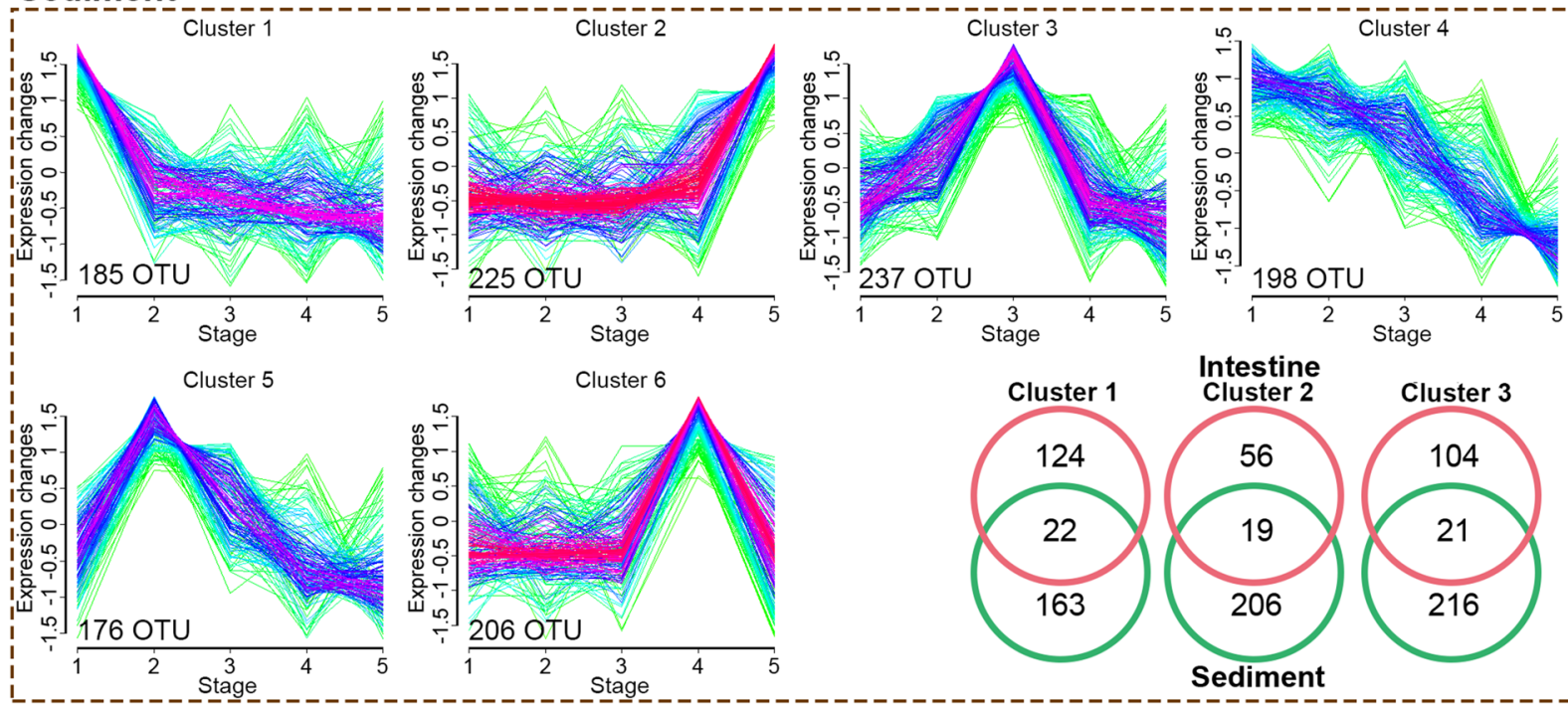

Sediment 
Table 2 PERMANOVA of microbial structure at different stages

\begin{tabular}{|c|c|c|c|c|c|c|c|}
\hline Habitat & Stage & 1 & 2 & 3 & 4 & 5 & $R^{2}$ \\
\hline \multirow[t]{5}{*}{ Water } & 1 & - & & & & & 0.215 \\
\hline & 2 & $0.01^{* *}$ & - & & & & \\
\hline & 3 & $0.03^{*}$ & 0.18 & - & & & \\
\hline & 4 & $0.01^{* *}$ & $0.03^{*}$ & 0.12 & - & & \\
\hline & 5 & $0.01^{* *}$ & $0.01^{* *}$ & $0.01^{* *}$ & $0.01^{* *}$ & - & \\
\hline \multirow[t]{5}{*}{ Intestine } & 1 & - & & & & & 0.278 \\
\hline & 2 & $0.01^{* *}$ & - & & & & \\
\hline & 3 & $0.01^{* *}$ & 0.1 & - & & & \\
\hline & 4 & $0.01^{* *}$ & $0.01^{* *}$ & $0.02^{*}$ & - & & \\
\hline & 5 & $0.01^{* *}$ & $0.01^{* *}$ & $0.01^{* *}$ & $0.01^{* *}$ & - & \\
\hline \multirow[t]{5}{*}{ Sediment } & 1 & - & & & & & 0.337 \\
\hline & 2 & 0.53 & - & & & & \\
\hline & 3 & 0.06 & 0.41 & - & & & \\
\hline & 4 & $0.01^{* *}$ & $0.01^{* *}$ & $0.01^{* *}$ & - & & \\
\hline & 5 & $0.01^{* *}$ & $0.01^{* *}$ & $0.01^{* *}$ & $0.02^{*}$ & - & \\
\hline
\end{tabular}

PERMANOVA was used to compare significant differences at different culture stages

Significant differences are indicated by asterisks $\left({ }^{*}, P<0.05 ;{ }^{*}, P<0.01\right)$

Microbial diversity, particularly, intestinal diversity, may be considered a feature of host health (Ren et al. 2018). In this study, the $\alpha$-diversity (Shannon index and Chao1 index) deceased during the culture period (Fig. 2). In a previous study, shrimp intestinal microbial diversity altered significantly at different culture stages (Zeng et al. 2017). Similar results have been observed in the intestinal microbiota of aquaculture fishes where the $\alpha$-diversity decreases over culture period (Yan et al. 2016). Another study suggested that larval shrimp can obtain sufficient and diverse bacterial species rapidly from the environment, although only a little part of the microbes could successfully colonize in the shrimp intestine (Xiong et al. 2018a). Some studies reveal that the development of animal digestive and immune systems may significantly affect the intestinal microbiota (Costello et al. 2012; Yan et al. 2016). The larvae, without complete immunity, may easily obtain more species from the environment, whereas the adult shrimp can selectively acquire different types of microbes. This finding is consistent with the processes found in other aquatic animals wherein microbial diversity decreases with host development (Burns et al. 2016; Yan et al. 2016). This information could be important for further microbial management for a more beneficial intestinal microbiota in shrimp culture, which might assist shrimp in preventing opportunistic pathogen or disease outbreaks.

Many investigations demonstrated that the important roles of intestinal microbial composition in aquatic animal health (Hou et al. 2018a; Li et al. 2017a, 2016; Xiong et al. 2018b). In addition, the intestine may host opportunistic pathogens, and it is evidenced that the overgrowth of opportunistic pathogens could contribute to disease (Perez et al. 2010; Xing et al. 2013). For example, in our previous study, the overgrowth of Candidatus Bacilloplasma and Phascolarctobacterium may contribute to white feces syndrome (Hou et al. 2018a). Similarly, Vibrio, Aeromonas and Photobacterium are generally known to include main opportunistic bacteria that could be pathogenic to aquatic animals (Nayak 2010; Zhang et al. 2014). Herein, the abundance of some opportunistic pathogens was relatively low (Aeromonas, $0.5 ; 10.7 \%$; Phascolarctobacterium, $<0.01 \%$; Photobacterium, 0.4\%;), whereas Vibrio (10.9\%) and Candidatus Bacilloplasma (10.4\%) were the dominant genera in shrimp intestine. This phenomenon was also detected in another study ( $\mathrm{Li}$ et al. 2017a), which suggested that these genera may act not only as opportunistic pathogens, but also as essential players with other unknown functions in the intestine environment. In addition, some probiotics were added during the culture period. However, the relative abundance of Bacillus was low in the three habitats $(<0.001 \%)$, which indicated that the use of probiotics did not effectively establish a large population in intestine and environment as expected. Unlike Bacillus, another probiotic Lactococcus was higher than $0.1 \%$ proportion in shrimp intestine. These results provide valuable clues for guiding the rational use of probiotics in shrimp culture.

Collectively, the composition and diversity of shrimp intestine microbiota and surrounding microbial community in Aquamimicry system were evaluated, with 
further investigation on the relationship between intestinal microbiota and the surrounding water and sediment. The microbial diversity of intestine and surroundings was different in Aquamimicry system, which indicates that dissimilarity among the three habitats could be a potential indictor for the healthy situation of shrimp culture, and will give us guidance for the sustainable shrimp culture practice to effectively promote the shrimp production. These findings may strengthen our understanding of the significance of microbial community in Aquamimicry system, and offer fundamental information to improve healthy culture practices in shrimp farming.

\section{Supplementary information}

Supplementary information accompanies this paper at https://doi. org/10.1186/s13568-020-01119-y.

Additional file 1: Table S1. Average shrimp weight performance every 15 days (g). Table S2. Average shrimp length performance every 15 days (cm). Figure S1. Microbial composition of water, intestine and sediment habitats at phylum level. Figure S2. Microbial composition of water, intestine and sediment habitats at genus level. Figure S3. Effect of culture stages and water physical chemical factors on microbial community.

\section{Acknowledgements}

This research was supported by the School of life sciences, Sun Yat-sen University (Guangzhou, China); Baxel Company Limited, Thailand and Lomrak Farm, Nakhon-Nayok province, Thailand. We would like to thank Lomrak farm staff (Nakhon-Nayok, Thailand) for the experimental samples, and the Department of Fishery Biology, Faculty of Fisheries, Kasetsart University, Thailand, for the laboratory analysis.

\section{Authors' contributions}

$\mathrm{ZH}, \mathrm{JH}$ and KS conceived this study. SZ, SK and ZH developed the experimental strategies and sampling design. SZ, SK, WK, DW, LY, ZX, HW and SW performed the sample collections, water quality determination, nucleotide extraction and sequencing data analysis. SZ, JH, ZH and KS wrote the manuscript. All authors read and approved the final manuscript.

\section{Funding}

This research was funded by the China Agriculture Research System (CARS-48); China-ASEAN Maritime Cooperation Fund, China-ASEAN Center for Joint Research and Promotion of Marine Aquaculture Technology; China National Postdoctoral Program for Innovative Talents (BX20200392); Guangdong MEPP Fund (NO.GDOE (2019) A21); Key research and development projects in Guangdong Province (2020B0202010009); Guangzhou Science Technology and Innovation Commission Project (201510010071); Guangdong Ocean and Fishery Bureau Project (20164200042090023).

\section{Availability of data and materials}

The 165 rRNA gene sequencing data and metagenomic data used in this study are available in the NCBI Short Read Archive (https://www.ncbi.nlm.nih. gov/sra) under Bioproject PRJNA625419.

Ethics approval and consent to participate

Not applicable.

\section{Consent for publication}

Not applicable.

\section{Competing interests}

The authors declare that they have no competing interests.

\section{Author details}

${ }^{1}$ State Key Laboratory of Biocontrol/Southern Marine Sciences and Engineering Guangdong Laboratory (Zhuhai), School of Marine Sciences, Sun Yat-Sen University, Guangzhou, China. ${ }^{2}$ Department of Fisheries, Faculty of Fisheries, Kasetsart University, Bangkok, Thailand. ${ }^{3}$ Institute of Aquatic Economic Animals and Guangdong Province Key Laboratory for Aquatic Economic Animals, School of Life Sciences, Sun Yat-Sen University, Guangzhou, China. ${ }^{4}$ Guangdong Provincial Key Laboratory of Marine Resources and Coastal Engineering, School of Marine Sciences, Sun Yat-Sen University, Guangzhou, China.

Received: 23 August 2020 Accepted: 24 September 2020

Published online: 06 October 2020

\section{References}

Al-Harbi AH, Uddin N (2005) Bacterial diversity of tilapia (Oreochromis niloticus) cultured in brackish water in Saudi Arabia. Aquaculture 250(3):566-572. https://doi.org/10.1016/j.aquaculture.2005.01.026

Amir S, Amy RS, Margaret K, Janelle B, Shawn M, Polly W, Robert L (2008) Aquaculture practices and potential human health risks: Current knowledge and future priorities. Environ Int 34(8):1215-1226. https://doi. org/10.1016/j.envint.2008.04.009

Anderson MJ (2006) Distance-based tests for homogeneity of multivariate dispersions. Biometrics 62(1):245-253. https://doi.org/10.111 1/j.1541-0420.2005.00440.x

Biesebeke R (2018) Balancing microbial ecosystems within humans and animals to prevent medical conditions. J Nutr Food Technol 1(2):40. https ://doi.org/10.30881/jnfrt.00009

Burns AR, Stephens WZ, Stagaman K, Wong S, Rawls JF, Guillemin K, Bohannan BJ (2016) Contribution of neutral processes to the assembly of gut microbial communities in the zebrafish over host development. ISME J 10(3):655-664. https://doi.org/10.1038/ismej.2015.142

Butto LF, Haller D (2016) Dysbiosis in intestinal inflammation: cause or consequence. Int J Med Microbiol 306(5):302-309. https://doi.org/10.1016/j. ijmm.2016.02.010

Caporaso JG, Kuczynski J, Stombaugh J, Bittinger K, Bushman FD, Costello EK, Fierer N, Peña AG, Goodrich JK, Gordon Jl, Huttley GA, Kelley ST, Knights D, Koenig JE, Ley RE, Lozupone CA, McDonald D, Muegge BD, Pirrung M, Reeder J, Sevinsky JR, Turnbaugh PJ, Walters WA, Widmann J, Yatsunenko T, Zaneveld J, Knight R (2010) QIIME allows analysis of highthroughput community sequencing data. Nat Methods 7:335. https://doi. org/10.1038/nmeth.f.303

Costello EK, Stagaman K, Dethlefsen L, Bohannan BJ, Relman DA (2012) The application of ecological theory toward an understanding of the human microbiome. Science 336(6086):1255-1262. https://doi.org/10.1126/scien ce. 1224203

Edgar RC (2010) Search and clustering orders of magnitude faster than BLAST. Bioinformatics 26(19):2460-2461. https://doi.org/10.1093/bioinformatics/ btq461

Edgar RC (2013) UPARSE: highly accurate OTU sequences from microbial amplicon reads. Nat Methods 10(10):996-998. https://doi.org/10.1038/ nmeth.2604

Flegel TW (2019) A future vision for disease control in shrimp aquaculture. J World Aquacult Soc 50(2):249-266. https://doi.org/10.1111/jwas.1258

Hou D, Huang Z, Zeng S, Liu J, Wei D, Deng X, Weng S, Yan Q, He J (2018a) Intestinal bacterial signatures of white feces syndrome in shrimp. Appl Microbiol Biotechnol 102(8):3701-3709. https://doi.org/10.1007/s0025 3-018-8855-2

Hou D, Huang Z, Zeng S, Liu J, Weng S, He J (2018b) Comparative analysis of the bacterial community compositions of the shrimp intestine, surrounding water and sediment. J Appl Microbiol 125(3):792-799. https://doi. org/10.1111/jam.13919

Huang Z, Zeng S, Xiong J, Hou D, Zhou R, Xing C, Wei D, Deng X, Yu L, Wang H, Deng Z, Weng S, Kriengkrai S, Ning D, Zhou J, He J (2020) Microecological Koch's postulates reveal that intestinal microbiota dysbiosis contributes to shrimp white feces syndrome. Microbiome 8:32. https://doi. org/10.1186/s40168-020-00802-3 
Huang Z, Chen Y, Weng S, Lu X, Zhong L, Fan W, Chen X, Zhang H, He J (2016) Multiple bacteria species were involved in hepatopancreas necrosis syndrome (HPNS) of Litopenaeus vannamei. Acta Sci Natur Univ SunYatseni 55(1):1-11. https://doi.org/10.13471/j.cnki.acta.snus

Israel JW, Martik ML, Byrne M, Raff EC, Raff RA, McClay DR, Wray GA (2016) Comparative developmental transcriptomics reveals rewiring of a highly conserved gene regulatory network during a major life history switch in the sea urchin genus Heliocidaris. PLoS Biol 14(3):e1002391. https://doi. org/10.1371/journal.pbio.1002391

Li T, Li H, Gatesoupe FJ, She R, Lin Q, Yan X, Li J, Li X (2017a) Bacterial signatures of "red-operculum" disease in the gut of crucian carp (Carassius auratus) Microb Ecol 74(3):510-521. https://doi.org/10.1007/s00248-017-0967-1

Li TT, Long M, Ji C, Shen ZX, Gatesoupe FJ, Zhang XJ, Zhang QQ, Zhang LL, Zhao YL, Liu XH, Li AH (2016) Alterations of the gut microbiome of largemouth bronze gudgeon (Coreius guichenoti) suffering from furunculosis. Sci Rep 6:9. https://doi.org/10.1038/srep30606

Li X, Zhou L, Yu Y, Ni J, Xu W, Yan Q (2017b) Composition of gut microbiota in the gibel carp (Carassius auratus gibelio) varies with host development. Microb Ecol 74(1):239-249. https://doi.org/10.1007/s00248-016-0924-4

Low CF, Rozaini MZH, MusaN SNB (2017) Current knowledge of metabolomic approach in infectious fish disease studies. J Fish Dis 40:1267-1277. https ://doi.org/10.1111/jfd.12610

Magoč T, Salzberg SL (2011) FLASH: fast length adjustment of short reads to improve genome assemblies. Bioinformatics 27(21):2957-2963. https:// doi.org/10.1093/bioinformatics/btr507

Nayak SK (2010) Role of gastrointestinal microbiota in fish. Aquacult Res 41(11):1553-1573. https://doi.org/10.1111/j.1365-2109.2010.02546.x

Perez T, Balcazar JL, Ruiz-Zarzuela I, Halaihel N, Vendrell D, de Blas I, Muzquiz JL (2010) Host-microbiota interactions within the fish intestinal ecosystem. Mucosal Immunol 3(4):355-360. https://doi.org/10.1038/mi.2010.12

Ren Z, Li A, Jiang J, Zhou L, Yu Z, Lu H, Xie H, Chen X, Shao L, Zhang R, Xu S, Zhang H, Cui G, Chen X, Sun R, Wen H, Lerut JP, Kan Q, Li L, Zheng S (2018) Gut microbiome analysis as a tool towards targeted non-invasive biomarkers for early hepatocellular carcinoma. Gut 68:1014-1023. https:// doi.org/10.1136/gutjnl-2017-315084

Schloss PD, Westcott SL, Ryabin T, Hall JR, Hartmann M, Hollister EB, Lesniewski RA, Oakley BB, Parks DH, Robinson CJ, Sahl JW, Stres B, Thallinger GG, Van Horn DJ, Weber CF (2009) Introducing mothur: open-source, platform-independent, community-supported software for describing and comparing microbial communities. Appl Microbiol Biotechnol 75(23):7537-7541. https://doi.org/10.1128/AEM.01541-09

Tassanakajon A, Rimphanitchayakit V, Visetnan S, Amparyup P, Somboonwiwat K, Charoensapsri W, Tang S (2018) Shrimp humoral responses against pathogens: antimicrobial peptides and melanization. Dev Comp Immunol 80:81-93. https://doi.org/10.1016/j.dci.2017.05.009

Xing MX, Hou ZH, Yuan JB, Liu Y, Qu YM, Liu B (2013) Taxonomic and functional metagenomic profiling of gastrointestinal tract microbiome of the farmed adult turbot (Scophthalmus maximus). FEMS Microbiol Ecol 86(3):432-443. https://doi.org/10.1111/1574-6941.12174

Xiong J, Dai W, Qiu Q, Zhu J, Yang W, Li C (2018a) Response of host-bacterial colonization in shrimp to developmental stage, environment and disease. Mol Ecol 27(18):3686-3699. https://doi.org/10.1111/mec.14822

Xiong J, Yu W, Dai W, Zhang J, Qiu Q, Ou C (2018b) Quantitative prediction of shrimp disease incidence via the profiles of gut eukaryotic microbiota. Appl Microbiol Biotechnol 102(7):3315-3326. https://doi.org/10.1007/ s00253-018-8874-Z

Xiong J, Xuan L, Yu W, Zhu J, Qiu Q, Chen J (2019) Spatiotemporal successions of shrimp gut microbial colonization: high consistency despite distinct species pool. Environ Microbiol 21(4):1383-1394. https://doi. org/10.1111/1462-2920.14578

Xiong J, Li X, Yan M, Lu J, Chen J (2020) Comparable ecological processes govern the temporal succession of gut bacteria and microeukaryotes as shrimp aged. Microb Ecol. https://doi.org/10.1007/s00248-020-01533-6

Yan Q, Li J, Yu Y, Wang J, He Z, Van Nostrand JD, Kempher ML, Wu L, Wang Y, Liao L, Li X, Wu S, Ni J, Wang C, Zhou J (2016) Environmental filtering decreases with fish development for the assembly of gut microbiota. Environ Microbiol 18(12):4739-4754. https://doi. org/10.1111/1462-2920.13365

Zeng S, Huang Z, Hou D, Liu J, Weng S, He J (2017) Composition, diversity and function of intestinal microbiota in pacific white shrimp (Litopenaeus vannamei) at different culture stages. PeerJ 5:e3986. https://doi.org/10.7717/ peerj.3986

Zeng S, Zhou R, Bao S, Li X, Deng Z, Hou D, Weng S, He J, Huang Z (2020) Identification of multigene biomarker for shrimp white feces syndrome by full-length transcriptome sequencing. Front Genet 11:71. https://doi. org/10.3389/fgene.2020.00071

Zhang M, Sun Y, Chen K, Yu N, Zhou Z, Chen L, Du Z, Li E (2014) Characterization of the intestinal microbiota in Pacific white shrimp, Litopenaeus vannamei, fed diets with different lipid sources. Aquaculture 434:449-455. https://doi.org/10.1016/j.aquaculture.2014.09.008

\section{Publisher's Note}

Springer Nature remains neutral with regard to jurisdictional claims in published maps and institutional affiliations.

\section{Submit your manuscript to a SpringerOpen ${ }^{\odot}$ journal and benefit from:}

- Convenient online submission

- Rigorous peer review

- Open access: articles freely available online

- High visibility within the field

- Retaining the copyright to your article

Submit your next manuscript at $\boldsymbol{\nabla}$ springeropen.com 